regions does not explain the fact that several hundred years ago the word Ada was a kingly title among the Jukon, the Kanuri, and the Southern Tuareg, whereas to-day it exists as the word for "father" in the case of the last named only, and that a variant form of $A d a$ (found in Arabic authors) as a title of the Mai of Bornu who introduced Islam about A.D. 1000 was Hadi which, considering all relevant data, can hardly be other than the Abyssinian title "Hati", which in turn was apparently the Meroitic title Ate.

'I should welcome correction or proof that I am in general mistaken in my thesis about the origins of Bornu and its cultural associations with both the Nile Valley and Southern Arabia, to which culturally Abyssinia in large measure belongs-a thesis which concerns the Tuareg of the Sahara as well as the Maghumi of Bornu.'

\title{
The Food and Nutrition of African Natives.
}

THE following Bibliography has been provided by Professor Labouret in connexion with his article entitled 'L'alimentation des autochtones dans les possessions tropicales' which appeared in the last number of this Journal. It supplements the information given in the bibliography contained in the Institute publication, The Food and Nutrition of African Natives (Memorandum XIII) in regard to studies made in French, Belgian, and Italian territories, and will thus be of special interest to a considerable number of readers.

\section{Colonies Françaises}

Burnet, Ét. : Alimentation en Tunisie. C. 7ז.

Gaud, Dr., et Sicault, Dr.: Alimentation indigène en Maroc. C. 8 I.

Berton, A. J.: Alimentation des indigènes d'Algérie. C. 63.

Martial, Dr. : Alimentation des indigènes en Afrique Occidentale Française.

Thiroux, A., Giroux, A., Ratsimamorga, R. : Alimentation des indigènes à Madagascar. C. 9 I.

Vassal, J. et Martial, J. E., Drs. : Alimentation des indigènes en Afrique Équatoriale Française. C. I 20.

VASSAL, Dr.: Alimentation en Indochine. C. 127.

Ces études sont contenues dans: La Science de l'Alimentation en 1937.

Labouret, H. : La lutte contre les famines et la disette en Afrique Tropicale. Afr. Fr., No. I1, Nov. 1937, No. I2, Déc. 1937.

— La géographie alimentaire en Afrique Occidentale, Extr. Annales de Géographie, Libr. Armand Colin, Paris, 1937.

\section{Congo Belge}

Bigwoon, E. J. : L'alimentation rationnelle de l'homme normal en Belgique, 1937, 59 pp. Publication du Ministère de la Santé Publique. Éd. de la croisade Nat. de Santé, 80 rue de Livourne, Bruxelles. 
BIGwoon, E. J. : Importance de l'alimentation rationnelle en hygiène sociale. XI ${ }^{\text {ème }}$ Congrès de l'CEuvre Nationale de l'Enfance, juin, 1937.

- Rapport sur les travaux du groupe des experts chargés d'étudier les méthodes d'appréciation de l'état de nutrition. Bull. Organ. d'Hyg. de la S.D.N., I937, vol. vi, extr. No. 5.

Ctautsr : Le Vème Congrès international d'Agriculture tropicale. Bull. Agric. C.B., 1930 .

_L Les sources d'alimentation des travailleurs indigènes: moyens de les développer. Bull. Agric. C.B., 1930.

Congrès d'A griculture 'Tropicale (V ème) 1930.

Congrès Colonial National, 1930.

De Wildeman, E.: Notes sur les plantes cultivées par les indigènes au Congo. Annales du Musée Colonial de Marseille, 1909.

— Le problème de l'alimentation de l'indigène au Congo Belge. Le Matériel colonial, 1937, xxvii, No. 5.

LeplaE: Notes au sujet du développement de l'agriculture du C.B. Tribune Congolaise, 5 avril 1917.

_Les cultures obligatoires dans les pays d'agriculture arriérée. Bull. Agric. C.B., 1929.

—_ Méthode suivie pour développer l'agriculture indigène au C.B. Bull. Agric. C.B., 1930.

- L'agriculture au Congo. Revue de l'Institut de Sociologie Solvay, 1932.

- Histoire et développement des cultures obligatoires. Revue Congo, 1932.

—- Paysannat intégral. Revue Congo, 1934.

Louwers: La question sociale au Congo. Revue Congo, i924.

Ministère des Colonies : Rapports Annuels du Congo Belge, I930 à 1936.

Rapports de la Commission de Protection des Indigk̀nes, 1919-1923.

Tinon, L.: A propos de quelques boissons fermentées indigènes. Bull. Agric. C.B., 1934, tome xxv.

Trolli et Bigwood: Problème de l'alimentation au Congo Belge. La Science de l'Alimentation en 1937, C. 4.43 .

\section{Colonies Italiennes}

Camrs, M.: Metabolismo basale ed alimentazione in Somalia. R. Acc. d'Italia, Roma, 1936.

Colletta, F.: Esperienze di alimentazione con semi di Lens abyssinica. Quad. Nutr., Napoli, 1936, t. iii, p. 301 .

Fantoli, A.: L'ambiente fisico delle colonie libiche nei suoi riflessi demografici, ecc. Rass. Econ. Coln., Roma, 1932, t. xx, p. 597.

LAPICQUE, L.: Étude quantitative sur le régime alimentaire des Abyssins. C.R. Soc. Biol., Paris, I 893, t. xlv.

Moruzzi, G., e TANzI, B.: Contributo alla conoscenza di alenni alimenti 
delle nostre colonie. Sul valore alimentare della pasta di datteri. Bioch. e Terap. Sperim., Milano, I933, t. xx.

Orru, A.: Analisi chimica e biologica di cereali africani. I. Sul valore alimentare delle cariossidi della Dagussa. Boll. Soc. It. Biol. Sper., Napoli, I929, t. iv.

Analisi chimica e biologica di cereali africani. III. Dati analitici sui principi immediati presenti nelle cariossidi di Eragrostis teff. Eleusine tocusso et Triticum durum. Boll. Soc. It. Biol. Sper., I93 1, t. vi.

_- Analisi chimica e biologica di cereali africani. IV. Il valore nutritivo delle cariossidi dell' Eragrostis teff. Boll. Soc. It. Biol. Sper., I 93 I, t. vi.

- Analisi chimica di semi di leguminose africane. I. Il valore biologico dei semi del cece amarico. Boll. Soc. It. Sper. 1930, t. v.

Analisi chimica e biologica di semi di leguminose africane. II. Dati analitici sui principi immediati presenti dei semi di: Cicer amaricum, Vigna catiang e Phaseolus mugo. Boll. Soc. It. Biol. Sper., I93 I, t. vi.

Esperienze di alimentazione con cariossidi di Dura Sorghum Aethiopicum. Quad. Nutr., Napoli, 1936, t. iii, p. 35 I.

Tedeschi, C., e Trotorano, L. : Nosografia della regione di Buloburti e di El Bur (Somalia Italiana). Arch. t. Sc. Med. Col. Parassitol., Modena, I934, t. Xv, pp. 86-III.

VARriale, R. : La campagna I9 2-I9I 3 della Squadriglia RR. Sambuchi dell' Oceano Indiano. Ann. Med. Nav., Roma, I9I4, t. xx, v, II, p. 368.

Vrsco, S.: Analisi chimica e biologica di cereali africani. II. Il valore nutritivo delle cariossidi del Pennisetum typhoïdeum. Boll. Soc. It. Biol. Sper., I930, t. v.

\section{A l'Institut Colonial International.'}

LEs sujets d'étude mis à l'ordre du jour par l'Institut Colonial International lors de ses dernières assises à Londres 1936 présentent pour les lecteurs d'Africa un intérêt tout particulier.

Les deux premiers rapports, introduits par des auteurs éminents et d'une compétence reconnue, concernent le statut des groupements indigènes constitués dans les centres européens ou à proximité de ces centres, l'un 'The Condition of Native Communities in or near European Centres', par le Major Orde Browne, l'autre 'Le Problème des centres extra-coutumiers et quelquesuns de ses aspects', par le R. P. Charles.

Nous ne résistons pas à l'envie de transcrire ici quelques-unes des thèses notamment les thèses ; , 6, 7, 8 et 9 - qui servent de résumé et de conclusion à l'exposé du R. P. Charles. 'Étant donné, dit-il, que ces centres et groupe-

I Bibliothèque Coloniale Internationale. Compte Rendu de la XXIIIe Session de 'Institut Colonial International tenue à Londres les $5,6,7$, et 8 octobre 1936 . Bruxelles, Établissements Généraux d'Imprimerie, 1937. 